\title{
Core-shell type thermo-nanoparticles loaded with temozolomide combined with photothermal therapy in melanoma cells
}

\author{
XIAOYANG HOU ${ }^{1}$, YANYU PANG ${ }^{1}$, XINXIN LI $^{1}$, CHUNSHENG YANG $^{2}$, \\ WENLOU LIU ${ }^{1}$, GUAN JIANG ${ }^{1}$ and YANQUN LIU ${ }^{1}$ \\ ${ }^{1}$ Department of Dermatology, Affiliated Hospital of Xuzhou Medical University, Xuzhou, Jiangsu 221002; \\ ${ }^{2}$ Department of Dermatology, The Affiliated Huai'an Hospital of Xuzhou Medical University, \\ The Second People's Hospital of Huai'an, Huai'an, Jiangsu 223002, P.R. China
}

Received May 9, 2019; Accepted August 23, 2019

DOI: $10.3892 /$ or.2019.7329

\begin{abstract}
A novel core-shell type thermo-nanoparticle (CSTNP) co-loaded with temozolomide (TMZ) and the fluorescein new indocyanine green dye IR820 (termed IT-CSTNPs) was designed and combined with a near-infrared (NIR) laser to realize its photothermal conversion. The IT-CSTNPs were prepared using a two-step synthesis method and comprised a thermosensitive shell and a biodegradable core. IR820 and TMZ were entrapped in the shell and the core, respectively. Dynamic light scattering results demonstrated that the average hydrodynamic size of the IT-CSTNPs was 196.4 $\pm 3.1 \mathrm{~nm}$ with a $\zeta$ potential of $-24.9 \pm 1.3 \mathrm{mV}$. The encapsulation efficiencies of TMZ and IR820 were 6.1 and $16.6 \%$, respectively. Temperature increase curves under NIR laser irradiation indicated that the IT-CSTNPs exhibited the desired photothermal conversion efficiency. The in vitro drug release curves revealed a suitable release capability of IT-CSTNP under physiological conditions, whereas NIR laser irradiation accelerated the drug release. Inverted fluorescence microscopy and flow cytometry results revealed that the uptake of IT-CSTNPs by A375 melanoma cells occurred in a concentration-dependent manner. Confocal laser scanning microscopy results indicated that IT-CSTNPs entered tumour cells via endocytosis and were located in intercellular lysosomes. In summary, the present study explored the photothermal conversion capability, cellular uptake, and intracellular localization of IT-CSTNPs.
\end{abstract}

\section{Introduction}

Melanoma is a malignant cancer originating from melanocytes, which often transforms from a single deteriorated

Correspondence to: Dr Yanqun Liu, Department of Dermatology, Affiliated Hospital of Xuzhou Medical University, 99 West Huaihai Road, Xuzhou, Jiangsu 221002, P.R. China

E-mail: 276512742@qq.com

Key words: melanoma, nanoparticles, photothermal therapy, temozolomide, IR820 melanocyte or a benign but dysfunctional mole (1). Melanoma is the deadliest skin malignancy, which tends to affect younger people, showing an alarming incidence that is increasing at $\sim 3 \%$ annually (2). Chemotherapy is an integral part of melanoma therapy. As a new alkylating agent that can be ingested orally, temozolomide (TMZ) features high bioavailability, wide tissue distribution, and can pass through the blood-brain barrier to reach an effective concentration in the central nervous system. TMZ is also recommended as an oral alternative to dacarbazine (DTIC) in many countries $(3,4)$. However, the drug resistance and instability of TMZ limit its therapeutic efficacy in melanoma. Thus, effective drug delivery has attracted increasing research attention.

Advances in nanotechnology have led to developments in the diagnosis and treatment of tumours (5). The biological structure and physicochemical properties of nanoparticles, such as small-size effect, surface functionality, multi-function effect and photo-magnetic properties, have prompted researchers to explore their biomedical applications $(6,7)$. The characteristics of nanoparticles, such as unique passive targeting effect and slow release, allow them to act as carriers to deliver drugs to the target site and achieve continuous drug release in tumours, effectively extending the half-life of drugs, while reducing their cytotoxicity to normal tissues (8). Although nano-delivery systems loaded with various chemotherapeutic drugs have achieved satisfactory results in the treatment of various cancers in recent decades, the monotherapy mode still features numerous deficiencies, such as poor drug release, requirement of multiple drug administration and poor efficacy. Photothermal therapy (PTT), as a promising alternative choice to traditional cancer therapies, uses photothermal conversion agents to destroy tumour cells by converting light into heat, and has attracted increasing attention (9). However, numerous problems limit the application of individual PPTs, including the difficulty of maintaining a constant temperature and duration, uneven heat distribution inside the tumour and incomplete tumour ablation. Therefore, the combination of PTT and chemotherapy in nanoparticles has recently attracted increasing attention (10).

To date, multiple nanocarriers, such as gold and carbon nanomaterials, have been used in combined thermo-chemotherapy $(11,12)$. However, given the poor degradability and high cost of such methods, researchers have focused on the 
biomedical application of near-infrared (NIR) dyes (13). For example, chemotherapeutic drugs and NIR dyes were encapsulated in nanoparticles, and photothermal conversion of NIR dyes was used to degrade the nanocarriers, in order to achieve 'controlled release' of the drugs $(14,15)$. The novel indocyanine green dye IR820, as a derivative of indocyanine green (ICG), possesses excellent stability and a longer tissue retention time than ICG $(16,17)$. Under NIR laser irradiation, IR820 can produce local heat and generate cytotoxic effects when the temperature exceeds the critical value $\left(42.5-43^{\circ} \mathrm{C}\right)(18)$. IR820 is easily metabolized by the liver in vivo. Thus, encapsulating IR820 in nanoparticles could improve its stability and local concentration in tumour sites. Li et al (19) constructed polymeric micelles loaded with the chemotherapy drug docetaxel (DTX) and IR820, and studied their combination treatment for breast cancer. With the help of nanocarriers, IR820 successfully accumulated in the tumour site. NIR laser irradiation increased the temperature of the tumour site and enhanced the sensitivity of cancer cells to DTX. Recent years have witnessed the rapid development of nanocarriers, such as polymeric nanoparticles, micelles, dendrimers and lipid nanocarriers. Core-shell lipid nanoparticles comprise a novel drug delivery system that includes a polymeric core and a phospholipid complex shell. Different drugs, genes or proteins can be loaded in the shell or core to achieve programmed drug release. In addition, core-shell lipid nanoparticles can also achieve simultaneous encapsulation of hydrophilic and hydrophobic drugs (20). The lipid shell is usually composed of lecithin, dipalmitoyl phosphatidylcholine (DPPC), and dioleoyl phosphoethanolamine. Polylactide-co-glycolic acid (PLGA), polycaprolactone, and dextran are the common biodegradable polymers used as the core of core-shell lipid nanoparticles (21). DPPC is the main component of thermosensitive liposomes, which can generate a gel-to-liquid phase transition in response to local hyperthermia $\left(41.5-41.9^{\circ} \mathrm{C}\right)$, thus triggering the release of a large amount of drug $(22,23)$. PLGA, approved by the U.S. Food and Drug Administration, is a promising biocompatible and degradable polymer that can be rapidly removed from the body, and is an ideal nanomaterial for drug delivery $(24,25)$. Li et al (26) designed a delivery system consisting of a DOX-modified PLGA core surrounded by a DPPC shell to treat breast cancer. Compared with free DOX, the delivery system exhibited a stronger antitumor effect at a lower concentration.

In the present study, core-shell type thermonanoparticles (CSTNPs) co-loaded with TMZ and IR820 (termed IT-CSTNPs) were developed as a potential chemical and photothermal combination therapy to treat melanoma. First, TMZ and IR820 were encapsulated in PLGA and DPPC to prepare PLGA-TMZ nanoparticles and IR820-DPPC lipid shells, respectively. In the next step, the IR820-DPPC lipid shells were used to coat the PLGA-TMZ nanoparticles in order to form the IT-CSTNPs. Finally, melanoma cells were exposed to the IT-CSTNPs and their uptake capacity and intracellular localization were investigated in vitro.

\section{Materials and methods}

Materials. PLGA was purchased from Xi'an Ruixi Biotechnology Co., Ltd. DPPC and monostearoylphosphatidylcholine (MSPC) were purchased from Avanti Polar Lipids Co.,
Ltd. IR820 and polyvinyl alcohol (PVA) were purchased from Sigma-Aldrich (Merck KGaA). TMZ was obtained from Tokyo Chemical Industry Co., Ltd. 4-6-Diamidino-2-phenylindole (DAPI) and coumarin-6 were purchased from Beijing Soleboard Biotechnology Co., Ltd. Lyso-Tracker Red was purchased from Shanghai Biyuntian Biotechnology Co., Ltd. Other solvents and reagents were purchased from Beijing Chemical Works.

Preparation of IT-CSTNPs. PLGA-TMZ nanoparticles were synthesized using double-emulsion solvent evaporation. First, $1 \mathrm{mg}$ of $\mathrm{TMZ}$ was dissolved in $\mathrm{HCl}(200 \mu \mathrm{l}, 0.1 \mathrm{M})$ to form an aqueous phase. Then, $10 \mathrm{mg}$ of PLGA was dissolved in $2 \mathrm{ml}$ of dichloromethane (DCM) to form an oil phase. The aqueous phase was added into the oil phase, and the obtained mixture was emulsified for $120 \mathrm{sec}$ using an ultrasonic processor to form the initial emulsion. Then, the emulsion was added into PVA $(1 \%, 10 \mathrm{ml})$ solution drop by drop and emulsified again. The homogeneous emulsion was stirred at room temperature overnight to devolatilize the DCM. PLGA-TMZ nanoparticles was collected by centrifugation at $16,654 \mathrm{x}$ g for $20 \mathrm{~min}$ at $4^{\circ} \mathrm{C}$ and washed three times with ultrapure water for subsequent use.

DPPC, MSPC, and IR820 (mass ratio, 14:1:1) were placed in a $50 \mathrm{ml}$ round-bottom flask with the addition of $5 \mathrm{ml}$ of chloroform/methanol (volume ratio, 4:1) solution to dissolve the lipids, and the organic solvent was removed using rotary evaporation under reduced pressure at $40^{\circ} \mathrm{C}$ to yield a thin lipid film. The film was subsequently hydrated with phosphate-buffered saline (PBS) solution of TMZ-NPs, followed by ultrasound for $5 \mathrm{~min}$. The spontaneously formed nanoparticles were extruded three times through 450 and $220 \mathrm{~nm}$ polyethersulfone ultrafiltration membranes, respectively. The unloaded drugs and lipids were removed by centrifugation at $10,281 \mathrm{x} g$ for $15 \mathrm{~min}$ at $4^{\circ} \mathrm{C}$. To assess the uptake and intracellular localization of nanoparticles, fluorescent coumarin-6-labeled IT-CSTNPs was prepared using the same process.

Characterization of IT-CSTNPs. The hydrodynamic size, polydispersity index (PDI), and $\zeta$ potential of the IT-CSTNPs were measured using dynamic light scattering (DLS) with a Zetasizer (Zetasizer Nano ZS; Malvern Instruments, Ltd.). The nanoparticle morphology was measured using transmission electron microscopy (TEM; FEI Tecnai G2 F20 U-TWIN; Thermo Fisher Scientific, Inc.). Briefly, one drop of IT-CSTNP suspension was deposited onto a carbon-coated copper grid, stained with $1 \%(\mathrm{w} / \mathrm{v})$ uranyl acetate for $1 \mathrm{~min}$, dried at room temperature, and observed by TEM.

TMZ and IR820 degradation curves. To accurately measure the TMZ content, the stability of TMZ was first determined in PBS at pH 7.4, in $0.1 \mathrm{M} \mathrm{HCl}$, and in dimethylformamide (DMF). TMZ solutions at $25 \mu \mathrm{g} / \mathrm{ml}$ were prepared with the corresponding solvents and then scanned in the wavelength range of 200-400 $\mathrm{nm}$ to obtain the ultraviolet absorption spectrum and determine the maximum absorption wavelength. The ultraviolet absorption curve was monitored at intervals $(0,1,2$, 4, 6 and $24 \mathrm{~h}$ ). The maximum absorption wavelength of IR820 was determined using the same method.

Encapsulation efficiency (EE) of IT-CSTNPs. Ultraspeed centrifugation at $10,281 \mathrm{x}$ g for $15 \mathrm{~min}$ at $4^{\circ} \mathrm{C}$ was used to 
separate the uncoated drugs, and the pellet and supernatant after each centrifugation were collected and subjected to demulsification with DMF. The absorbance of drugs in DMF was determined using an ultraviolet spectrophotometer.

The EE of the IT-CSTNPs was calculated as follows: EE $(\%)=(\mathrm{C} 1 \mathrm{xV} 1) /(\mathrm{C} 1 \mathrm{xV} 1+\mathrm{C} 2 \mathrm{xV} 2) \times 100$; where $\mathrm{V} 1$ and $\mathrm{V} 2$ refer to the volume of collected pellet and supernatant, respectively; and $\mathrm{C} 1$ and $\mathrm{C} 2$ are the drug concentrations of the collected pellet and supernatant, respectively, calculated according to the standard curve of ultraviolet absorption value-concentration.

Temperature increase curve under NIR laser irradiation. A NIR laser $\left(808 \mathrm{~nm}, 6 \mathrm{~W} / \mathrm{cm}^{2}\right)$ was used to irradiate the PBS solution (pH 7.4) containing IT-CSTNPs (IR820 concentrations, $0,5,10$ and $20 \mu \mathrm{g} / \mathrm{ml}$ ) for $5 \mathrm{~min}$ at an initial temperature of $24.7^{\circ} \mathrm{C}$. The temperature increase values of the IT-CSTNP solution at different time-points were recorded using an IR thermometer.

The PBS solution at pH 7.4 with or without $20 \mu \mathrm{g} / \mathrm{ml}$ of free IR820 and IT-CSTNPs (IR820 concentration, $20 \mu \mathrm{g} / \mathrm{ml}$ ) wereirradiated with NIR laserat $6 \mathrm{~W} / \mathrm{cm}^{2}$ for $4.75 \mathrm{~min}$.Temperature was recorded using an IR thermometer to plot the temperature increase curve.

In vitro TMZ release. The dialysis-bag method was used to investigate TMZ release under different $\mathrm{pH}$ conditions. A total of $700 \mu \mathrm{l}$ of IT-CSTNPs (TMZ content, $54 \mu \mathrm{g}$ ) were added into three identical dialysis bags (molecular weight cut-off, 8,000-14,000 Da). The two ends were tightened with thin wires and placed in three $50 \mathrm{ml}$ centrifuge tubes. Then, PBS solutions at pH 5.0, 6.5 and 7.4 were added into the centrifuge tubes separately. The centrifuge tubes were placed on a constant-temperature oscillator at $100 \mathrm{rpm}$ at $37^{\circ} \mathrm{C}$ to simulate the release of TMZ in vitro. After removing $1 \mathrm{ml}$ of the released solution at given intervals, $1 \mathrm{ml}$ of the corresponding fresh PBS was added to the centrifuge tubes. Absorption values of liquids at $329 \mathrm{~nm}$ and the concentration and cumulative drug release rate were calculated at corresponding time-points.

NIR-induced drug release in vitro was also investigated. After simulating drug release on a shaker for $30 \mathrm{~min}$, the IT-CSTNPs were aspirated from the dialysis bag and transferred to $1.5 \mathrm{ml}$ Eppendorf tubes. The tubes were then irradiated using a NIR laser $\left(6 \mathrm{~W} / \mathrm{cm}^{2}\right)$ for $5 \mathrm{~min}$ and transferred into the original dialysis bag. The simulated drug release was further performed in a thermostatic oscillator. At different time-points, $1 \mathrm{ml}$ of the release solution was obtained, and the absorption value was measured at $329 \mathrm{~nm}$. The concentration and cumulative drug release rate at corresponding time-points were calculated.

Cell culture. Human melanoma cells (A375) were purchased from the Type Culture Collection of the Chinese Academy of Sciences (Shanghai, China) and routinely grown in Dulbecco's modified Eagle's medium (DMEM; Gibco; Thermo Fisher Scientific, Inc.) supplemented with $10 \%$ foetal bovine serum (FBS) (Gibco; Thermo Fisher Scientific, Inc.), and $100 \mathrm{U} / \mathrm{ml}$ penicillin and $100 \mu \mathrm{g} / \mathrm{ml}$ streptomycin (Invitrogen; Thermo Fisher Scientific, Inc.). The cells were cultured in a $5 \% \mathrm{CO}_{2}$-saturated humidity incubator at $37^{\circ} \mathrm{C}$.
Cellular uptake analysis. Uptake of IT-CSTNPs at different concentrations by A375 cells was detected using an inverted fluorescence microscope and flow cytometry. A375 cells, at the logarithmic growth phase, were seeded in six-well plates at a density of $2 \times 10^{5}$ cells/well and cultured overnight. IT-CSTNPs labelled with coumarin-6 (IR820 concentrations, $0,5,10$ and $20 \mu \mathrm{g} / \mathrm{ml}$ ) were added to the culture medium. After incubation for $3 \mathrm{~h}$, the cells were washed three times with PBS and subsequently fixed with $4 \%$ paraformaldehyde for $15 \mathrm{~min}$ at room temperature. Then, the nucleus was stained with DAPI for $5 \mathrm{~min}$, and the cells were observed under an inverted fluorescence microscope. Flow cytometry was also used to measure the cellular uptake. A375 cells were incubated with IT-CSTNPs labelled with coumarin-6 (IR820 concentrations, $0,5,10$ and $20 \mu \mathrm{g} / \mathrm{ml}$ ) for $3 \mathrm{~h}$ and then digested with trypsin and collected by centrifugation $(300 \mathrm{x} \mathrm{g}, 5 \mathrm{~min})$ at room temperature. The cells were then washed with PBS and detected using a flow cytometer (BD Biosciences), followed by analysis on FlowJo v10 software (Tree Star, Inc.).

Intracellular localization analysis. A375 cells at the logarithmic growth phase were seeded in a confocal glass-bottom culture dish at a density of $1 \times 10^{5}$ cells and incubated overnight at $37^{\circ} \mathrm{C}$. IT-CSTNPs labelled with coumarin-6 (IR820, $20 \mu \mathrm{g} / \mathrm{ml}$ ) were added to the culture medium. After $3 \mathrm{~h}$ of incubation, the culture medium was discarded, and the cells were gently washed three times with PBS. Then, $1 \mathrm{ml}$ of Lyso-Tracker Red dye $(50 \mathrm{nM})$ medium was added to the dishes and incubated for $1 \mathrm{~h}$ at $37^{\circ} \mathrm{C}$. After washing the cells with PBS, images were captured under a laser confocal microscope.

Statistical analysis. All values are presented as the mean \pm standard deviation. The statistical significance of the differences between experimental groups was calculated using either unpaired Student's t-test or one-way ANOVA followed by Bonferroni post hoc test. $\mathrm{P}<0.05$ was considered to indicate a statistically significant difference.

\section{Results}

Preparation and characterization of IT-CSTNPs. The IT-CSTNPs were prepared using a two-step synthesis method. PLGA-TMZ nanoparticles were synthesized by the double-emulsion solvent evaporation method. IR820 was dispersed into DPPC to form a lipid film using thin-film dispersion. Then, the IT-CSTNPs were synthesized through hydration, ultrasonication and extrusion (Fig. 1). DLS was applied to characterize the particle size, PDI and $\zeta$ potential of the IT-CSTNPs. The results demonstrated that the average hydrodynamic size of an IT-CSTNP was about $196.4 \pm 3.1 \mathrm{~nm}$, the PDI was $0.181 \pm 0.03$, and the $\zeta$ potential was $-24.9 \pm 1.3 \mathrm{mV}$ (Fig. 2A and B). In addition, the particle size was measured in DMEM+10\% FBS using DLS, and the results demonstrated that the hydrodynamic size of the IT-CSTNPs was not significantly altered, indicating that the nanoparticles could maintain good integrity and stability in cell culture conditions (Fig. 2A). The morphology of the IT-CSTNPs was observed using TEM. The image revealed that the IT-CSTNPs were spherical, uniform in size and exhibited no significant agglomeration; a core-shell 

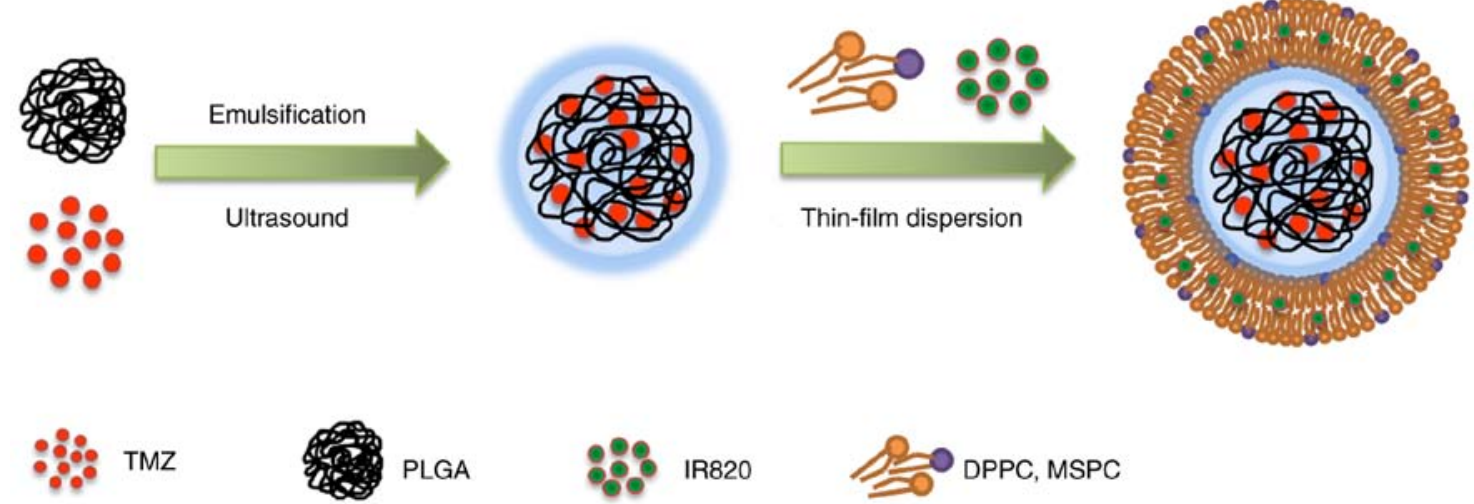

Figure 1. Schematic for preparation of core-shell type thermo-nanoparticles co-loaded with TMZ and the fluorescein new indocyanine green dye IR820. TMZ, temozolomide; PLGA, polylactide-co-glycolic acid; DPPC, dipalmitoyl phosphatidylcholine; MSPC, monostearoylphosphatidylcholine.

structure could be observed, indicating that the PLGA core was successfully surrounded by a lipid shell (Fig. 2C).

EEs of TMZ and IR820. Next, the UV absorption method was used to determine the content of TMZ and IR820. TMZ was unstable in PBS solution, and its maximum absorption peak at $329 \mathrm{~nm}$ decreased with time (Fig. 3A). However, the UV absorption curve of TMZ in $0.1 \mathrm{M} \mathrm{HCl}$ exhibited no significant change with time, and had a maximum absorption peak at $329 \mathrm{~nm}$, indicating that $0.1 \mathrm{M} \mathrm{HCl}$ could be used as the water phase in double-emulsion solvent evaporation to prevent TMZ degradation to a certain extent during the synthesis process (Fig. 3B). The maximum absorption wavelength and stability of TMZ was further investigated in DMF. The results demonstrated that TMZ exhibited the largest absorption peak at $329 \mathrm{~nm}$ and no degradation within $24 \mathrm{~h}$, suggesting that TMZ was stable in DMF solution; therefore, DMF could be used as the demulsification solvent (Fig. 3C). According to the aforementioned formula, the EEs of TMZ and IR820 were 6.1 and $16.6 \%$, respectively.

Temperature increase curve of the IT-CSTNPs. The increasing temperature capacities of IT-CSTNPs at different concentrations under NIR laser irradiation were measured, in order to detect their photothermal conversion capability. As presented in Fig. 4A, the temperature of the IT-CSTNP solution increased as the drug concentration increased under NIR laser irradiation. When the concentration of IT-CSTNPs was $10 \mu \mathrm{g} / \mathrm{ml}$, the maximum temperature was $4.8^{\circ} \mathrm{C}$, and at $20 \mu \mathrm{g} / \mathrm{ml}$ the maximum temperature was $8.6^{\circ} \mathrm{C}$. Furthermore, at the same IR820 concentration $(20 \mu \mathrm{g} / \mathrm{ml})$, the increased temperature of the IT-CSTNPs was slightly higher compared with that of free IR820 (Fig. 4B), indicating that the IT-CSTNPs possessed a better photothermal conversion capability than free IR820.

In vitro drug release profile. To examine the release properties of TMZ from IT-CSTNPs, a simulated drug release experiment was performed. Fig. 5A presents the drug release profile of IT-CSTNPs in PBS at various pH values. In PBS solutions $\mathrm{pH}$ 5.0, 6.5 and 7.4, the amount of TMZ released within $24 \mathrm{~h}$ reached 32.6, 47.4 and $46.9 \%$, respectively.

In addition, the release of TMZ from IT-CSTNPs was examined after NIR laser irradiation. The release curve in Fig. 5B demonstrates that drug release increased significantly
Table I. Uptake of IT-CSTNPs by A375 cells.

\begin{tabular}{lc}
\hline $\begin{array}{l}\text { Experimental } \\
\text { groups }\end{array}$ & $\begin{array}{c}\text { Average fluorescence } \\
\text { intensity (mean } \pm \text { SD) }\end{array}$ \\
\hline NP-0 (control) & $2746 \pm 79.9$ \\
NP-5 & $10733.33 \pm 1011.789^{\mathrm{a}}$ \\
NP-10 & $21122 \pm 3372.866^{\mathrm{a}, \mathrm{b}}$ \\
NP-20 & $28858.67 \pm 1825.35^{\mathrm{a}-\mathrm{c}}$ \\
\hline
\end{tabular}

NP-0, NP-5, NP-10 and NP-20 indicate that the concentrations of IT-CSTNPs were 0 (control group), 5, 10 and $20 \mu \mathrm{g} / \mathrm{ml}$, respectively. ${ }^{\mathrm{a} P}<0.05$ compared with the control group; ${ }^{\mathrm{b}} \mathrm{P}<0.05$ compared with NP-5 group; and ${ }^{\mathrm{C}} \mathrm{P}<0.05$ compared with NP-10 group $(\mathrm{n}=3)$. IT-CSTNPs, core-shell type thermo-nanoparticles co-loaded with temozolomide and IR820.

after $0.5 \mathrm{~h}$ of NIR laser irradiation; at $24 \mathrm{~h}, 56.3 \%$ of the TMZ was released in the $\mathrm{NIR}^{+}$group compared with only $34.1 \%$ in the $\mathrm{NIR}^{-}$group (Fig. 5B). These results indicated that the high temperature generated by NIR laser irradiation promoted the release of TMZ from the IT-CSTNPs.

Cellular uptake profile. Coumarin-6, a hydrophobic fluorescent molecule, was encapsulated in the core-shell nanoparticles, in order to label the whole nanoparticles and to trace their uptake and intracellular localization. The results of inverted fluorescence microscopy revealed that there was no green fluorescence distribution in the control group. After coumarin-6-labeled IT-CSTNP exposure, significant green fluorescence was observed in the A375 cells, and the fluorescence intensity correlated positively with the concentration of IT-CSTNPs, indicating that cellular internalization of IT-CSTNPs by A375 cells was concentration-dependent in the selected range.

The uptake of IT-CSTNPs was further quantified using flow cytometry. With the increase in drug concentration, the fluorescence signal of cells increased, exhibiting concentration dependence (Fig. 6B; Table I). The result was consistent with the results of inverted fluorescence microscopy.

Intracellular localization profile. Laser confocal microscopy images (Fig. 7) revealed the presence of substantial amounts of 

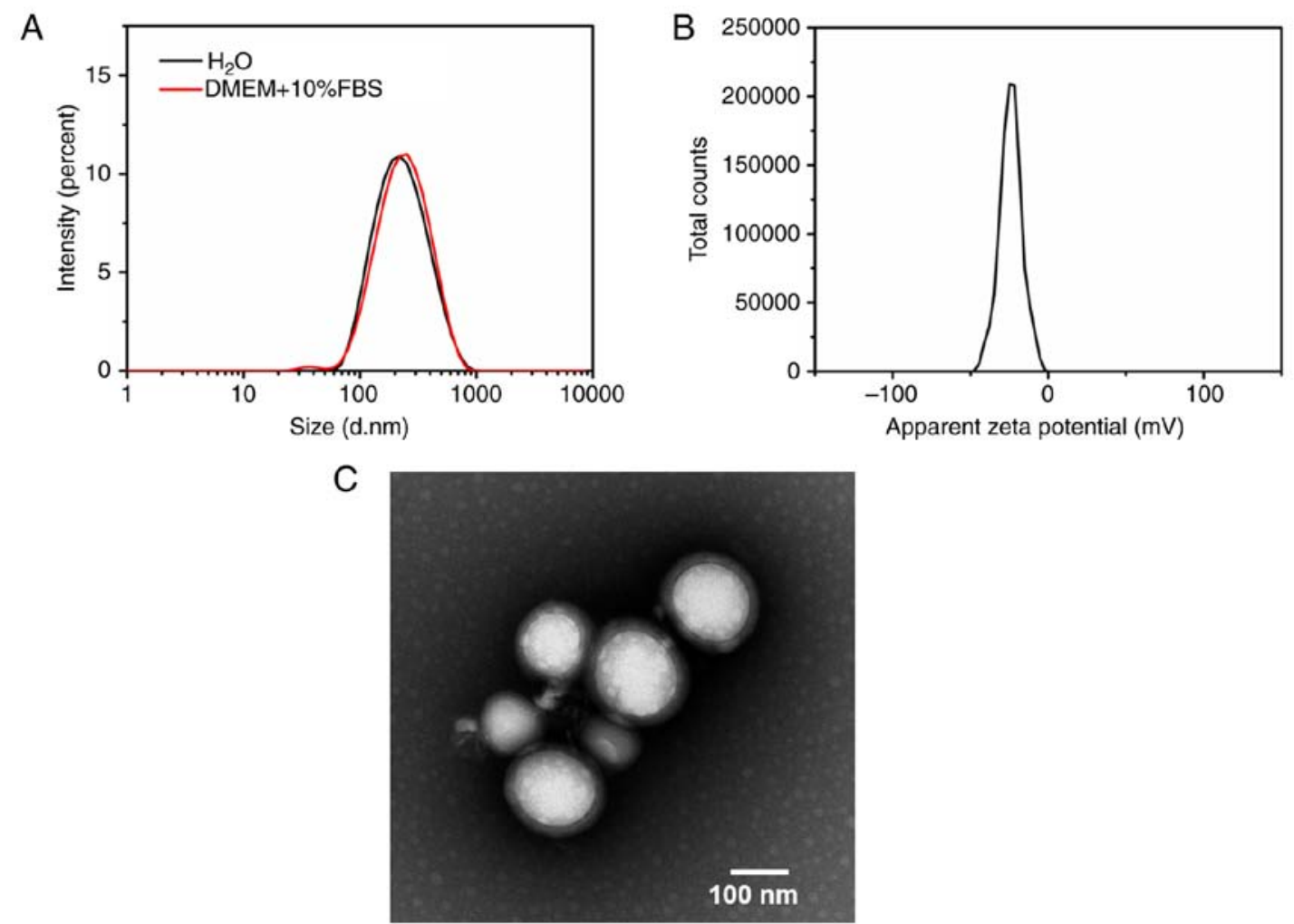

Figure 2. Characterization of core-shell type thermo-nanoparticles co-loaded with temozolomide and the fluorescein new indocyanine green dye IR820. (A) Hydrodynamic size distribution in water and in culture media (DMEM+10\% FBS). (B) $\zeta$ potential. (C) Transmission electron microscopy image.
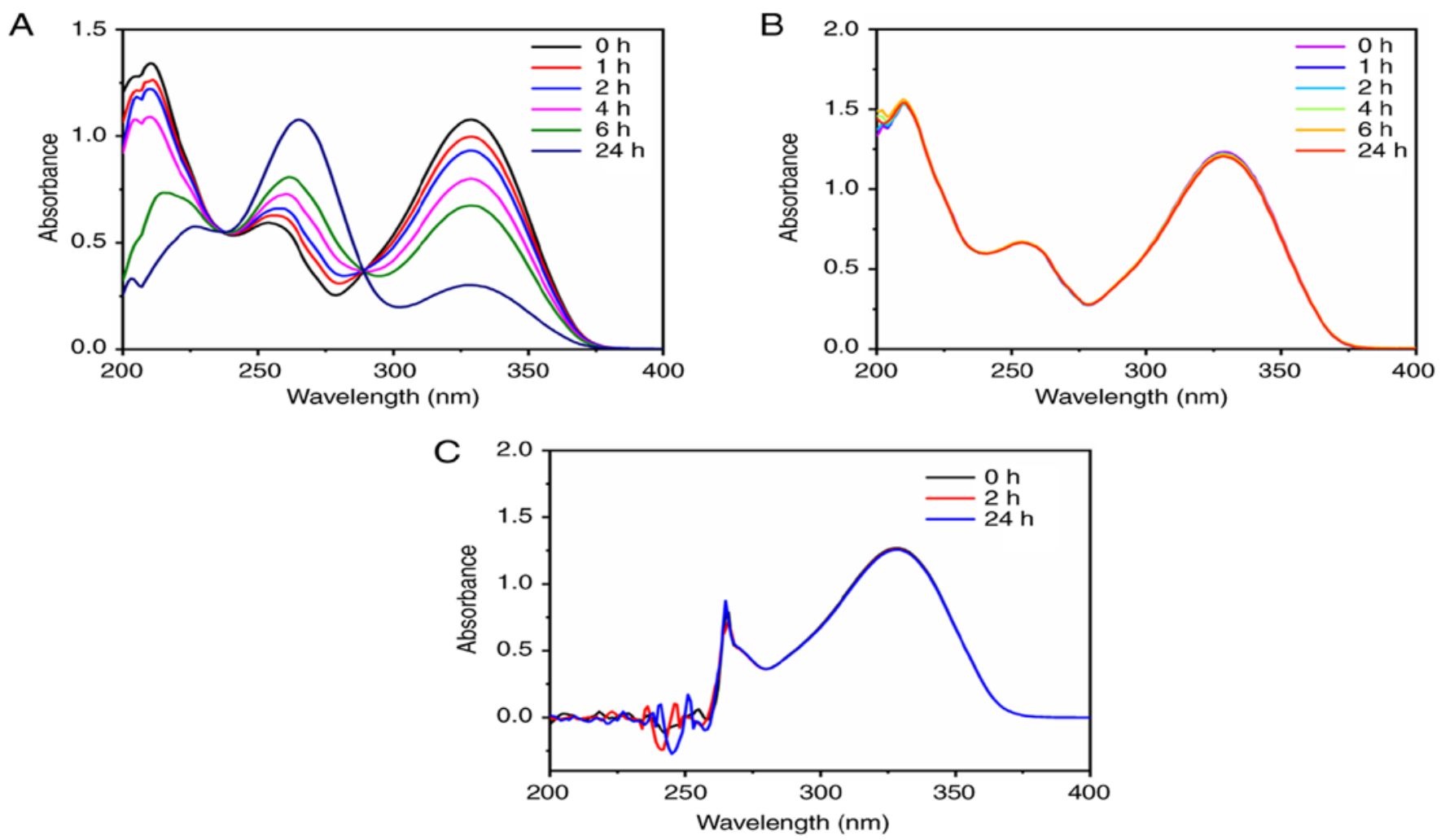

Figure 3. Determination of the TMZ content. (A) Degradation curve of TMZ in PBS pH 7.4. (B) Ultraviolet absorption curve of TMZ in 0.1 M HCl. (C) Ultraviolet absorption spectrum and stability of TMZ. TMZ, temozolomide.

green fluorescence in A375 cells, which was consistent with the aforementioned results of inverted fluorescence microscopy and flow cytometry (Fig. 6). After staining the lysosomes with the specific Lyso-Tracker Red dye, it was observed that the 

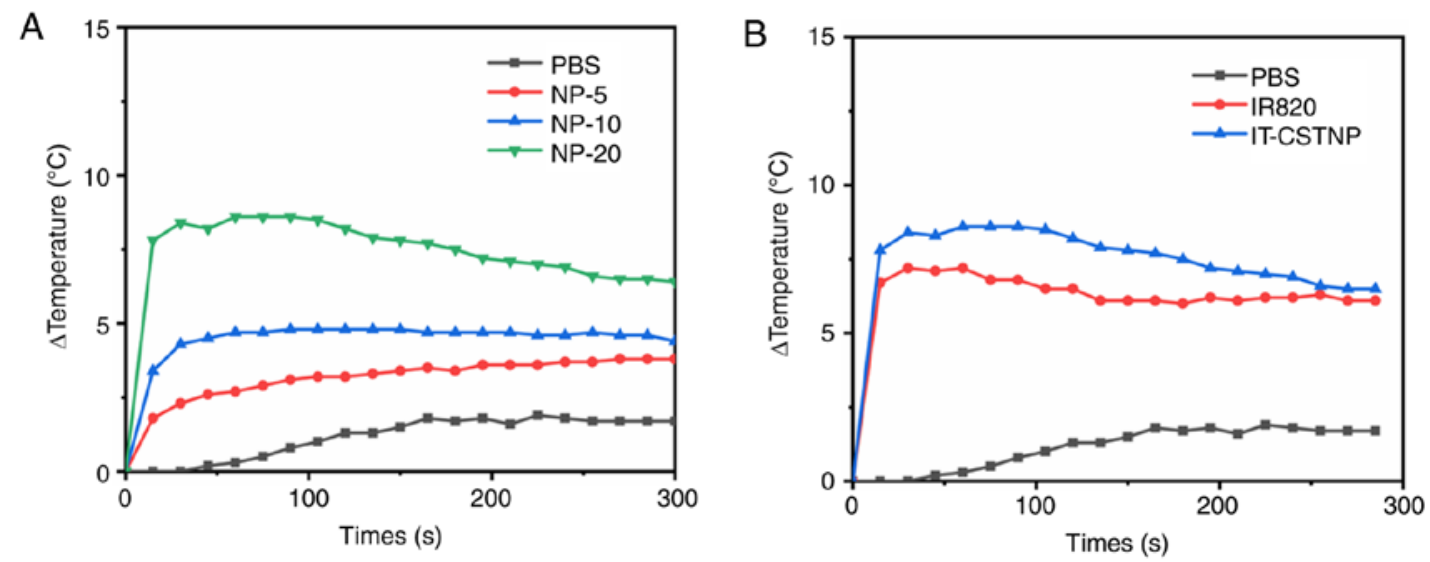

Figure 4. Temperature increase curve measurement. (A) Temperature increase curves of different concentrations of IT-CSTNPs $(0,5,10$ and $20 \mu \mathrm{g} / \mathrm{ml})$ under near-infrared laser irradiation. (B) Temperature curves of PBS, IR820 $(20 \mu \mathrm{g} / \mathrm{ml})$, and IT-CSTNPs (IR820 concentration, $20 \mu \mathrm{g} / \mathrm{ml})$ under near-infrared laser irradiation. IT-CSTNPs, core-shell type thermo-nanoparticle co-loaded with temozolomide and IR820; NP, nanoparticle.
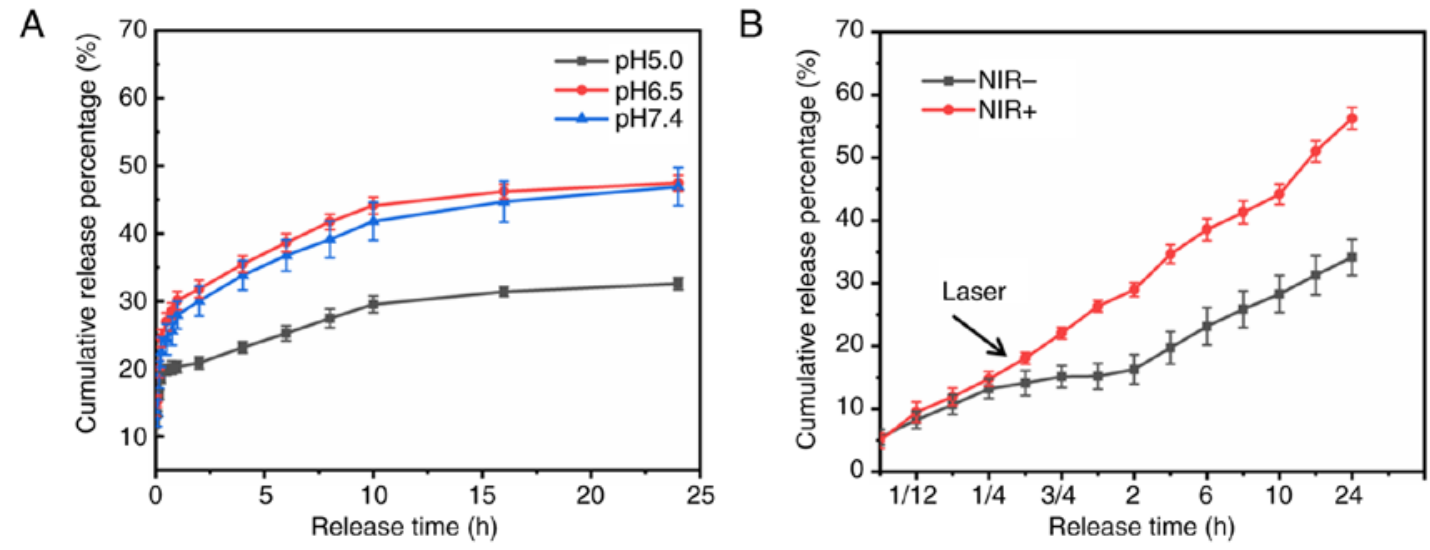

Figure 5. In vitro drug release profile of IT-CSTNPs. (A) Release profile of IT-CSTNPs at different pH conditions. (B) Release profile of IT-CSTNPs under NIR irradiation. IT-CSTNPs, core-shell type thermo-nanoparticle co-loaded with temozolomide and IR820; NIR, near-infrared.

IT-CSTNP signal (green) coincided with that of the lysosomes (red), indicating the entry of IT-CSTNPs into the lysosomes. Therefore, it was concluded that IT-CSTNPs entered the cells via endocytosis and were transported to lysosomes by endocytic vesicles.

\section{Discussion}

Melanoma is a malignant cancer with insidious onset and high invasiveness. In recent years, the incidence of melanoma has increased continually globally and is showing a trend of affecting a younger population, although its incidence in Asia is lower than that in Europe and the United States (2). Melanoma is one of the main causes of skin cancer-associated mortality. The median survival time of patients is 6-10 months, and the five-year survival rate is $<20 \%(27,28)$. Early diagnosis and surgical resection can improve the prognosis. Advanced melanoma progresses rapidly and is prone to metastasis. Radiotherapy, chemotherapy, immunotherapy and targeted therapy can be used to treat melanoma. However, conventional treatments, such as radiotherapy and chemotherapy, have very limited effects. TMZ, as a new second-generation oral alkylating agent, has advantages, such as high bioavailability, wide tissue distribution, the capability to pass the blood-brain barrier and a favourable therapeutic effect to treat brain metastatic melanoma. TMZ is recommended in numerous countries as an oral replacement for DTIC. However, several problems such as drug resistance and side effects still limit TMZ's application and development. Therefore, there is an urgent need to develop effective, safe and non-toxic anti-melanoma drugs.

In recent years, the rapid development of nanomedicine has provided new insights into the challenges faced by melanoma treatment. Nanoparticles have been used widely in biomedical fields as highly effective and targeted drug carriers. The increased vascular permeability of the tumour tissue and dysfunction of lymphatic system reflux lead to an enhanced permeation retention (EPR) effect, causing nanoparticles in the range of $10-500 \mathrm{~nm}$ to easily aggregate at the tumour site, representing the 'passive targeting effect' of nanoparticles. Thus, drugs encapsulated in nanoparticles can be retained at the tumour site by the EPR effect, and an effective concentration can be reached to yield the optimal therapeutic effect while reducing systemic toxicity $(27,29)$. With continued research progress, the design focus of nanoparticles is gradually moving toward more complex core-shell structures using a single nano-delivery system to combine multiple functions of 
A

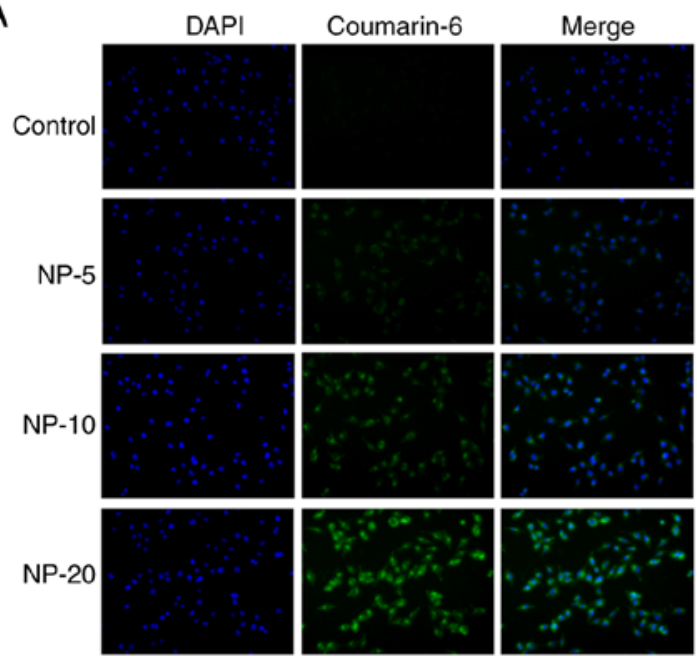

B

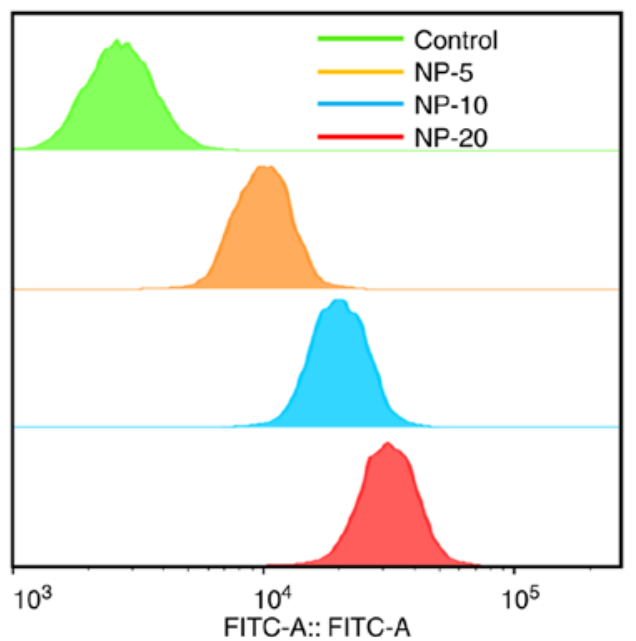

Figure 6. Cellular uptake of IT-CSTNPs. Dose-dependent cellular uptake of IT-CSTNPs by A375 melanoma cells was measured using (A) inverted fluorescence microscopy (magnification, x200) and (B) flow cytometry. IT-CSTNPs, core-shell type thermo-nanoparticle co-loaded with temozolomide and IR820; FITC, fluorescein isothiocyanate; NP, nanoparticle.
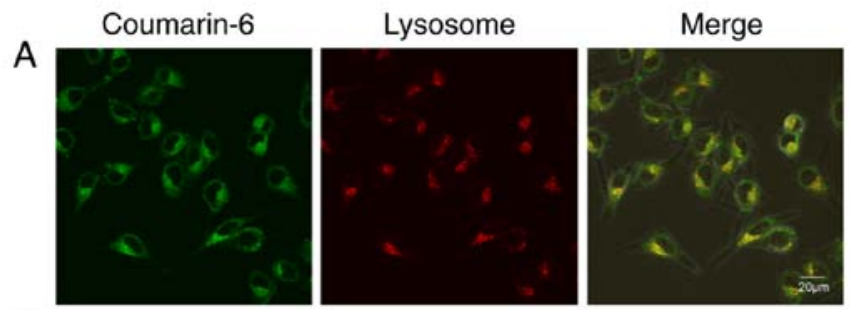

B
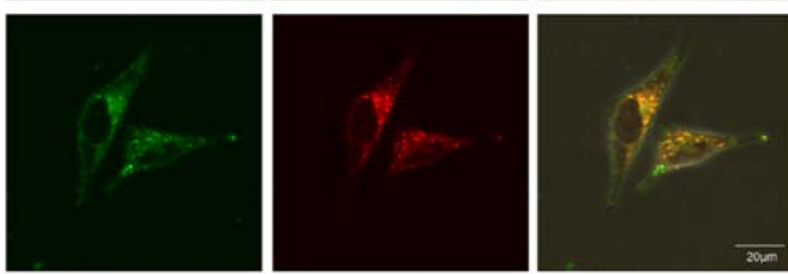

Figure 7. Intracellular localization measurement of core-shell type thermo-nanoparticles co-loaded with temozolomide and the fluorescein new indocyanine green dye IR820 in A375 cells. (A) and (B) Represent different magnifications. Scale bar, $20 \mu \mathrm{m}$.

different nanoparticles. Lipid polymer hybrid nanoparticles are favoured by researchers because they combine the structural stability of polymers with the biofilm compatibility of lipid shells (21). Lipid polymer hybrid nanoparticles contain at least two components: A polymer and a lipid. PLGA is often used as the polymeric core because of its good biodegradability and biocompatibility. DPPC is used as the lipid shell to encapsulate PLGA to form a cell-like structure. Simple PLGA nanoparticles are easily removed from blood circulation via opsonization, and the exposed PLGA encounters difficulty in entering cells. The lipid layer can serve as a molecular fence to protect the PLGA core and improve nanoparticle absorption by tumour cells (30).

In the present study, the TEM results revealed that IT-CSTNPs comprising DPPC lipids and PLGA polymers were successfully synthesized. The DLS results indicated that the IT-CSTNPs were 196.4 $\pm 3.1 \mathrm{~nm}$ in size, uniform, and monodispersed, benefitting the passive targeting of tumour sites via the EPR effect.
Although TMZ is listed as a first-line treatment for malignant tumours in many countries, it is easily degradable under physiological $\mathrm{pH}$ conditions, resulting in changes in the ultraviolet absorption wavelength and thereby increasing the difficulty of characterization. TMZ is more stable in an acidic environment; therefore, $0.1 \mathrm{M} \mathrm{HCl}$ was used as the aqueous phase. The present study used a lipid shell and polymer core to encapsulate IR820 and TMZ and to synthesize IT-CSTNPs, and explored their photothermal conversion capability under NIR laser irradiation. The EEs of TMZ and IR820 measured via the ultraviolet absorption method were 6.1 and $16.6 \%$, respectively. The EE of TMZ was consistent with the results of Ananta et al (31), whereas that of IR820 was slightly lower than the $19.8 \%$ reported by $\mathrm{Wu}$ et al (16). Such results can be possibly attributed to the poor solubility of TMZ in water, which made it difficult for TMZ to embed in PLGA, whereas excess lipids could adsorb the drug to form vesicles and be lost during hydration and extrusion. In addition, the 'two-step synthesis' preparation method caused the IR820 to release the lipid shell on the nanoparticle surface, and this condition might be accompanied by the release of the drug from the film, thereby affecting their EE values (32).

PTT is a safe and effective non-invasive treatment that has been widely used in cancer treatment. Under irradiation by an external light source, PTT uses the thermal effect of photothermal conversion agents to convert light energy into heat; thus, the temperature of the tumour site is raised to an effective treatment temperature, thereby achieving the effect of directly or indirectly killing the tumour cells $(33,34)$. The temperature increase curve generated in the present study revealed that under NIR irradiation, and when the IT-CSTNPs concentration was $20 \mu \mathrm{g} / \mathrm{ml}$, the highest temperature rise could reach $8.6^{\circ} \mathrm{C}$. If the initial temperature was $37.5^{\circ} \mathrm{C}$, the maximum temperature could thus reach $46.1^{\circ} \mathrm{C}$, and at a concentration of $10 \mu \mathrm{g} / \mathrm{ml}$, the maximum temperature that could be reached would be $42.3^{\circ} \mathrm{C}$, which is within the moderate thermal therapy range $\left(41-43^{\circ} \mathrm{C}\right)$. This temperature not only inhibits the growth of tumour cells, but also facilitates drug release. In addition, 
the present results demonstrated that the ability of IT-CSTNPs to increase the temperature at the same IR820 concentration was better compared with that of free IR820, which might be caused by the accumulation of nanoparticles.

The results of NIR laser-induced drug release experiments showed that, compared with the control group, the $\mathrm{NIR}^{+}$group released significantly more of the drug, indicating that when the temperature increased to the phase transition temperature, DPPC generated a gel-to-liquid phase transition, thereby realizing the 'burst release' of the drug. In addition, the $\mathrm{pH}$ gradient release curve showed no significant difference in the percentage of release from IT-CSTNPs in PBS solution at $\mathrm{pH} 6.5$ and 7.4 within $24 \mathrm{~h}$ (47.4 and $46.9 \%$, respectively), with both values being greater than that observed at pH 5.0 (32.6\%). Possibly because the nanoparticles are negatively charged, a large amount of $\mathrm{H}^{+}$present in the acidic environment wraps around them, resulting in the slow release of TMZ.

The main challenge of traditional chemotherapy for cancer is multidrug resistance; a predominant mechanism for this is thought to occur through the activity of the transmembrane protein $\mathrm{P}$-glycoprotein ( $\mathrm{P}-\mathrm{gp})$, which can transfer drugs out of tumour cells (35). Drug-loaded nanoparticles can enter tumour cells by endocytosis and be transported by endocytic vesicles to lysosomes, thereby avoiding being pumped out by P-gp (36). The results of flow cytometry and inverted fluorescence microscopy revealed obvious green fluorescence in the cells after incubation with IT-CSTNPs for $3 \mathrm{~h}$; the uptake of IT-CSTNPs in the different concentration groups was significantly higher compared with that in the control group $(\mathrm{P}<0.05$; Table $\mathrm{I})$ and increased with increasing concentration $(\mathrm{P}<0.05$; Table $\mathrm{I})$. The results of laser confocal microscopy showed that the signals for IT-CSTNPs (green) and for the lysosomes (red) were fused, indicating that the nanoparticles colocalized with the lysosomes, suggesting that IT-CSTNPs entered cells via endocytosis and concentrated in the lysosomes.

In conclusion, the present study successfully synthesized IT-CSTNPs with simultaneous loading of the chemotherapy drugs TMZ and NIR dye IR820, and explored their photothermal conversion capability, cellular uptake and intracellular localization. The current findings are expected to provide new directions for melanoma treatment.

\section{Acknowledgements}

Not applicable.

\section{Funding}

This research was supported by the National Natural Science Foundation of China (grant nos. 81572976, 81872493 and 81803151), the China Postdoctoral Science Foundation (grant nos. 2016M590505 and 2017T100407), the Jiangsu Provincial Medical Talent Foundation, the 'Six Talent Peaks' Project of Jiangsu Province (grant nos. WSW-074 and WSN-254), the Science and Technology Project of Huai'an city (grant no. HAB201812), and the Innovation of Graduate Student Training Projects in Jiangsu Province of China (grant no. KYCX18_2187).

\section{Availability of data and materials}

The datasets supporting the conclusions of this article are included within this article.

\section{Authors' contributions}

YL and XH designed the study. XH, YP, XL, CY and WL performed the experiments and analysed the data. XH, YP and XL wrote the manuscript. YL and GJ helped to revise the manuscript. All authors have read and approved the final manuscript.

\section{Ethics approval and consent to participate}

Not applicable.

\section{Patient consent for publication}

Not applicable.

\section{Competing interests}

The authors declare that they have no competing interests.

\section{References}

1. Damsky WE and Bosenberg M: Melanocytic nevi and melanoma: Unraveling a complex relationship. Oncogene 36: 5771-5792, 2017.

2. Tripp MK, Watson M, Balk SJ, Swetter SM and Gershenwald JE: State of the science on prevention and screening to reduce melanoma incidence and mortality: The time is now. CA Cancer J Clin 66: 460-480, 2016.

3. Chiarion-Sileni V, Guida M, Ridolfi L, Romanini A, Del Bianco P, Pigozzo J, Brugnara S, Colucci G, Ridolfi R, De Salvo GL and Italian Melanoma Intergroup (IMI): Central nervous system failure in melanoma patients: Results of a randomised, multicentre phase 3 study of temozolomide- and dacarbazine-based regimens. Br J Cancer 104: 1816-1821, 2011.

4. Kim C, Lee CW, Kovacic L, Shah A, Klasa R and Savage KJ: Long-term survival in patients with metastatic melanoma treated with DTIC or temozolomide. Oncologist 15: 765-771, 2010.

5. Jahangirian H, Kalantari K, Izadiyan Z, Rafiee-Moghaddam R, Shameli $\mathrm{K}$ and Webster TJ: A review of small molecules and drug delivery applications using gold and iron nanoparticles. Int J Nanomedicine 14: 1633-1657, 2019.

6. Jindal AB: The effect of particle shape on cellular interaction and drug delivery applications of micro- and nanoparticles. Int J Pharm 532: 450-465, 2017.

7. Hoshyar N, Gray S, Han H and Bao G: The effect of nanoparticle size on in vivo pharmacokinetics and cellular interaction. Nanomedicine (Lond) 11: 673-692, 2016.

8. Samanta D, Meiser JL and Zare RN: Polypyrrole nanoparticles for tunable, $\mathrm{pH}$-sensitive and sustained drug release. Nanoscale 7: 9497-9504, 2015.

9. Sheng W, He S, Seare WJ and Almutairi A: Review of the progress toward achieving heat confinement-the holy grail of photothermal therapy. J Biom Opt 22: 80901, 2017.

10. Zhang X, Du J, Guo Z, Yu J, Gao Q, Yin W, Zhu S, Gu Z and Zhao Y: Efficient near infrared light triggered nitric oxide release nanocomposites for sensitizing mild photothermal therapy. Adv Sci (Weinh) 6: 1801122, 2018.

11. D'Acunto M: Detection of intracellular gold nanoparticles: An overview. Materials (Basel) 11: E882, 2018.

12. Doughty ACV, Hoover AR, Layton E, Murray CK, Howard EW and Chen WR: Nanomaterial applications in photothermal therapy for cancer. Materials (Basel) 12: E779, 2019.

13. Kim H, Chung K, Lee S, Kim DH and Lee H: Near-infrared light-responsive nanomaterials for cancer theranostics. Wiley Interdiscip Rev Nanomed Nanobiotechnol 8: 23-45, 2016. 
14. Zhou B, Li Y, Niu G, Lan M, Jia Q and Liang Q: Near-infrared organic dye-based nanoagent for the photothermal therapy of cancer. ACS Appl Mater Interfaces 8: 29899-29905, 2016.

15. Chen Y, Chen Q, Zhu Q, Liu J, Li Y, Gao X, Chen D and Zhu X: Small-molecular theranostic assemblies functionalized by doxorubicin-hyaluronic acid-methotrexate prodrug for multiple tumor targeting and imaging-guided combined chemo-photothermal therapy. Mol Pharm 16: 2470-2480, 2019.

16. Wu B, Wan B, Lu ST, Deng K, Li XQ, Wu BL, Li YS, Liao RF, Huang SW and Xu HB: Near-infrared light-triggered theranostics for tumor-specific enhanced multimodal imaging and photothermal therapy. Int J Nanomed 12: 4467-4478, 2017.

17. Hu X, Tian H, Jiang W, Song A, Li Z and Luan Y: Rational design of IR820- and ce6-based versatile micelle for single NIR laser-induced imaging and dual-modal Phototherapy. Small 14: e1802994, 2018.

18. Hildebrandt B, Wust P, Ahlers O, Dieing A, Sreenivasa G, Kerner T, Felix R and Riess H: The cellular and molecular basis of hyperthermia. Crit Rev Oncol Hematol 43: 33-56, 2002.

19. Li W, Peng J, Tan L, Wu J, Shi K, Qu Y, Wei X and Qian Z: Mild photothermal therapy/photodynamic therapy/chemotherapy of breast cancer by Lyp-1 modified Docetaxel/IR820 Co-loaded micelles. Biomaterials 106: 119-133, 2016.

20. Mukherjee A, Waters AK, Kalyan P, Achrol AS, Kesari S and Yenugonda VM: Lipid-polymer hybrid nanoparticles as a next-generation drug delivery platform: State of the art, emerging technologies, and perspectives. Int J Nanomedicine 14 1937-1952, 2019.

21. Mandal B, Bhattacharjee H, Mittal N, Sah H, Balabathula P Thoma LA and Wood GC: Core-shell-type lipid-polymer hybrid nanoparticles as a drug delivery platform. Nanomedicine 9: 474-491, 2013.

22. de Matos MBC, Beztsinna N, Heyder C, Fens MHAM, Mastrobattista E, Schiffelers RM, Leneweit G and Kok RJ: Thermosensitive liposomes for triggered release of cytotoxic proteins. Eur J Pharm Biopharm 132: 211-221, 2018.

23. Turner DC, Moshkelani D, Shemesh CS, Luc D and Zhang H: Near-infrared image-guided delivery and controlled release using optimized thermosensitive liposomes. Pharm Res 29: 2092-2103, 2012

24. Tong L, Liao Q, Zhao Y, Huang H, Gao A, Zhang W, Gao X, Wei W, Guan M, Chu PK and Wang H: Near-infrared light control of bone regeneration with biodegradable photothermal osteoimplant. Biomaterials 193: 1-11, 2019.

25. Albert C, Huang N, Tsapis N, Geiger S, Rosilio V, Mekhloufi G, Chapron D, Robin B, Beladjine M,Nicolas V, et al: Bare and sterically stabilizedPLGA nanoparticles for the stabilization of pickering emulsions. Langmuir 34: 13935-13945, 2018.
26. Li B, Xu H, Li Z, Yao M, Xie M, Shen H, Shen S, Wang X and Jin Y: Bypassing multidrug resistance in human breast cancer cells with lipid/polymer particle assemblies. Int J Nanomedicine 7: 187-197, 2012.

27. Chen J, Shao R, Zhang XD and Chen C: Applications of nanotechnology for melanoma treatment, diagnosis, and theranostics. Int J Nanomedicine 8: 2677-2688, 2013.

28. Silva IP and Long GV: Systemic therapy in advanced melanoma: Integrating targeted therapy and immunotherapy into clinical practice. Curr Opin Oncol 29: 484-492, 2017.

29. Yingchoncharoen P, Kalinowski DS and Richardson DR Lipid-Based drug delivery systems in cancer therapy: What is available and what is yet to come. Pharmacol Rev 68: 701-787, 2016.

30. Sah H, Thoma LA, Desu HR, Sah E and Wood GC: Concepts and practices used to develop functional PLGA-based nanoparticulate systems. Int J Nanomedicine 8: 747-765, 2013.

31. Ananta JS, Paulmurugan R and Massoud TF: Temozolomideloaded PLGA nanoparticles to treat glioblastoma cells: A biophysical and cell culture evaluation. Neurol Res 38: 51-59, 2016.

32. Cheow WS and Hadinoto K: Factors affecting drug encapsulation and stability of lipid-polymer hybrid nanoparticles. Colloids Surf B Biointerfaces 85: 214-220, 2011.

33. Chen WR, Singhal AK, Liu H and Nordquist RE: Antitumor immunity induced by laser immunotherapy and its adoptive transfer. Cancer Res 61: 459-461, 2001.

34. Hu Y, Chi C, Wang S, Wang L, Liang P, Liu F, Shang W, Wang W, Zhang F, Li S, et al: A comparative study of clinical intervention and interventional photothermal therapy for pancreatic cancer. Adv Mater 29: 2017.

35. Hunyadi A, Csabi J, Martins A, Molnar J, Balazs A and Toth G: Backstabbing P-gp: Side-chain cleaved ecdysteroid 2,3-dioxolanes hyper-sensitize MDR cancer cells to doxorubicin without efflux inhibition 22: E199, 2017.

36. Chen HH, Lu IL, Liu TI, Tsai YC, Chiang WH, Lin SC and Chiu HC: Indocyanine green/doxorubicin-encapsulated functionalized nanoparticles for effective combination therapy against human MDR breast cancer. Colloids Surf B Biointerfaces 177: 294-305, 2019.

This work is licensed under a Creative Commons Attribution-NonCommercial-NoDerivatives 4.0 International (CC BY-NC-ND 4.0) License. 